\title{
Diferencias de edad y género en el uso y consumo de medios sociales entre los adolescentes
}

\author{
Age and gender differences in use and \\ consumption of social media among adolescents
}

Antonio García-Jiménez Universidad Rey Juan Carlos

Beatriz Catalina-García Universidad Rey Juan

Victoria Tur-Viñes Universidad de Alicante

\section{Referencia de este artículo}

García-Jiménez, Antonio; Catalina-García, Beatriz y Tur-Viñes, Victoria (2021). Diferencias de edad y género en el uso y consumo de medios sociales entre los adolescentes. En: adComunica. Revista Científica de Estrategias, Tendencias e Innovación en Comunicación, $\mathrm{n}^{\circ} 22$. Castellón de la Plana: Departamento de Ciencias de la Comunicación de la Universitat Jaume I, 211-234. DOI: http://dx.doi. org/10.6035/2174-0992.2021.22.12

\section{Palabras clave}

Adolescentes, consumo, medios sociales, Instagram, YouTube, redes sociales.

\section{Keywords}

Teenagers, consumption, social media, Instagram, YouTube, social networks. 


\title{
Resumen
}

Este artículo aborda el comportamiento y actividades que realizan los adolescentes en las redes sociales como parte de su consumo mediático. En la literatura previa sobre el tema se observa la prevalencia de estudios enfocados en los riesgos y vulnerabilidades digitales (consecuencias) en detrimento de las actividades y usos (tipo de consumo) de este grupo de edad. Este estudio complementa lo existente, abordando la finalidad del consumo de los adolescentes en cada red social. Las actividades analizadas están relacionadas con la autopresentación, las relaciones sociales, el entretenimiento, la búsqueda de diferentes tipos de contenidos, y el contacto con las noticias. Con una encuesta aplicada a una muestra representativa ( $\mathrm{N}=515)$ de adolescentes entre 12 y 16 años, se determina la relación entre las variables de edad y género y el tipo de uso y consumo. Para la mayoría de actividades, el uso de Instagram presenta un crecimiento progresivo con la edad, hasta los 16 años donde cae su uso. YouTube presenta la tendencia inversa. En cuanto al género, se observan diferencias en las actividades de autopresentación y en el seguimiento de las actualizaciones de familia y amigos. En general, la relevancia de la imagen y de los intereses personales queda reflejada.

\begin{abstract}
This article addresses the behaviour and activities of adolescents on social networks as part of their media use. Previous literature on the subject points to the prevalence of studies focused on digital risk and vulnerability (consequences) to the detriment of activity and use (type of consumption) of this age group. This study contributes to the existing literature, addressing the final objective that adolescents have in consuming each of the social networks. The activities analysed are related to self-presentation, social relations, the entertainment, the search for different types of content, and the contact with the news. Through the use of a survey conducted with a representative sample $(\mathrm{N}=515)$ of adolescents between 12 and 16 years of age, the aim of this study is to determine the influence exerted on social network use according to the type of use and the variables of age and gender. For most of the activities under consideration, the use of Instagram increases progressively until the age of 16, when its use declines. YouTube shows the opposite trend. In terms of gender, differences in self-presentation activities and in following updates from family and friends are observed. In general, the relevance of image and personal interests has been detected.
\end{abstract}

\section{Autores}

Antonio García-Jiménez [antonio.garcia@urjc.es] es Catedrático de Periodismo de la URJC (2017-). Ha sido Decano de la Facultad de Ciencias de la Comunicación de la URJC (2008-2014). Investigador principal del grupo de investigación GICOM- 
SOC, presenta una gran producción tanto de artículos, libros y capítulos de libro. Sus áreas de interés son: adolescentes y jóvenes en internet y la Documentación en los medios de comunicación.

Beatriz Catalina-García [beatriz.catalina@urjc.es] es Doctora en Ciencias de la Comunicación por la URJC (2011), licenciada en Periodismo (Universidad Complutense, 1989) y licenciada en Ciencias Políticas (UNED, 2014). Profesora Contratada Doctor en la URJC. Ha participado en diversos proyectos de investigación relacionados con usos de Internet y Nuevas Tecnologías. Sus líneas de investigación se centran en Audiencias, Esfera Pública Digital y Comunicación en Internet.

Victoria Tur-Viñes [victoria.tur@gcloud.ua.es] es Catedrática del Departamento de Comunicación y Psicología Social de la Universidad de Alicante. Docente del Grado en Publicidad y Relaciones Públicas en creatividad publicitaria. Directora departamento (2009-2013). Coordinadora del Máster en Comunicación e Industrias Creativas (2010-20). Tiene reconocidos 2 sexenios de investigación y es autora de más de 80 publicaciones: 7 libros, 25 capítulos y 40 artículos científicos indexados. 


\section{Introducción ${ }^{1}$}

Este estudio pertenece a la investigación centrada en el uso y consumo de los medios sociales. En concreto, se estudia el uso y consumo de plataformas de redes sociales por parte de adolescentes de 12 a 16 años conforme a las actividades que llevan a cabo teniendo en cuenta la edad y el género.

Varios modelos se proponen para analizar este objeto de estudio. Uno de los más importantes es el referido a los comportamientos de riesgo, especialmente enfocados a niños y adolescentes (De Vries, Peter, et al., 2016; Gini, Marino, et al., 2019; Scott y Cleeland-Woods, 2018; Vannucci, Simpson, et al., 2020; Young, Len-Ríos y Young, 2017). Otros estudios analizan las motivaciones de uso de los medios sociales, basados en gran medida en la Teoría de Usos y Gratificaciones (Al-Menayes, 2015; Throuvala, Griffiths, et al., 2019; Trültzsch-Wijnen, TrültzschWijnen y Siibak, 2015). Un tercer grupo de estudios se centran en otros aspectos como las tareas que realizan los individuos en los medios y los medios sociales, (Twenge, Martin y Spitzberg, 2019), la participación cívica de los jóvenes en los medios digitales (van Eldik, Kneer y Jansz, 2019), o la consulta de noticias (Bergstrom y Jervelycke, 2018).

En la línea de Throuvala, Griffiths, et al. (2019), definimos a los medios sociales como servicios electrónicos en Internet que permiten crear y compartir contenidos. Engloban blogs, sitios o plataformas de redes sociales, microblogs, sitios para compartir contenidos o para los videojuegos. En muchos artículos, se adopta, entre otros, el término «social media» como similar al de «social networking site». Cuando se haga referencia a Instagram, YouTube, Facebook, Snapchat o Twitter, optamos por los términos medio social y plataforma de red social.

\subsection{Usos de medios sociales y actividades}

Diferentes estudios muestran los rápidos cambios que los menores desarrollan en un acceso al entorno digital que, además, es cada vez más intenso. Como caso particular, Twenge, Martin y Spitzberg (2019) hallan que el 82\% de los estadounidenses de entre 13 y 18 años consulta diariamente las redes sociales, porcentaje que apenas llegaba un 50\% hace poco más de una década, y acceden al mundo en línea entre 4 y 6 horas diarias, una frecuencia que les resta tiempo para los medios tradicionales: en general, ven menos la televisión y sólo el 16\% reconoce que lee diariamente un libro o una revista. A pesar de que unos años antes, Len-Ríos, Hughes, et al. (2015) registran que los adolescentes entre 12 y 14 años pasaban más tiempo ante el televisor que con las redes sociales, ya se observaba una creciente multitarea digital y multiplicidad de perfiles sociales.

1 Créditos de la investigación: Proyecto $\mathrm{I}+\mathrm{D}+\mathrm{i}$ Redes sociales, adolescentes y jóvenes: convergencia de medios y cultura digital (CSO2016-74980-C2-2-R). 
En el caso particular de España, se ha pasado en poco tiempo de Tuenti a Facebook y después a Instagram (Marcelino Mercedes, 2015), con una tímida inmersión en Music.Ly — ahora Tik Tok - y sobre todo en YouTube (Pérez Escoda, 2018). Igualmente, se detectan transformaciones en el comportamiento y en los usos a medida que se hacen mayores. En este contexto y relacionado con los usos, el consumo de vídeos es, según Tejada, Castaño y Romero (2019), la actividad más frecuente entre menores de 10 y 12 años. Un 87\% accede a Internet para ver vídeos, hacer trabajos académicos $(80,9 \%)$, buscar información $(72,6 \%)$ o enviar mensajes $(61 \%)$.

A esas edades, las redes sociales no forman parte aún de sus preferencias. Apenas el 30\% las visita regularmente, aunque las más populares son YouTube e Instagram (a pesar de que la autorización para acceder a esta última es de 14 años). Entre usuarios de redes algo más mayores -de 14 a 17-prefieren WhatsApp y expresan su indiferencia con Facebook (Cívico Ariza, González García y Colomo Magaña, 2019). Instagram consigue el reconocimiento de los usuarios con menor edad por su adaptación para la producción, edición y difusión de fotografías mediante la propia cámara del teléfono (Candale, 2017).

Fernández de Arroyabe, Lazkano y Eguskiza (2018) constatan la preeminencia de Instagram, pero también de YouTube para compartir contenidos audiovisuales. La elección depende del alcance deseado para la difusión y tipo de contenido: ambas redes si es público e Instagram si es privado (81\%). Igualmente observan diferencias de uso en función del género: las mujeres interactúan intensamente, consumen más YouTube y ven videoclips, mientras que los chicos se decantan por las videoconsolas. Ellas consumen más información de todas las temáticas que ellos a excepción de la deportiva.

En este empleo de los medios sociales, Van Dam y Van Rejimersdal (2019) detectan una gran aceptación de lo publicado por los influencers. Los adolescentes observan una actitud positiva, incluso honesta, en los contenidos patrocinados y recuerdan más las marcas publicitadas por las celebridades que las promocionadas por desconocidos (Murphy, Corcoran, et al., 2020). Entre los adolescentes españoles de 12 a 18 años, solo un 4,3\% usan Instagram para seguir cuentas relacionadas con noticias. Prefieren los memes, la música, la moda y los deportes (Gil-Quintana y Fernández-Galiano, 2020).

Las actividades diarias más frecuentes entre menores de 9 a 16 años son ver videoclips y escuchar música, y realizar mensajes instantáneos. En el siguiente orden, alrededor de un tercio busca información en el entorno digital, hacen las tareas escolares con la ayuda de Internet y visitan algún perfil en las redes sociales. Los usos que más se han incrementado son la participación en videojuegos, el consumo de videos y la publicación de mensajes. La frecuencia de estas actividades aumenta a medida que se van acercando a la mayoría de edad (Garmendia, Jiménez, et al., 2016).

Más recientemente, se observa que los hábitos de uso varían sensiblemente. En efecto, el estudio sobre menores de Eukids Online aplicado al ámbito español 
(Garmendia, Jiménez, et al., 2019) muestra que los usos diarios más recurrentes por los menores se vinculan a la comunicación con su entorno y, en segundo lugar, los relacionados con el entretenimiento, principalmente la escucha de música (63\%), la visualización de videoclips (55\%) y, jugar online (46\%), más agudizado esta actividad entre los chicos. También lo utilizan para las actividades escolares, sobre todo ellas. Con una frecuencia menor, que oscila en torno a una quinta parte de los adolescentes, se sitúa la compra online (22\%) y la búsqueda online de noticias (19\%). Todas estas actividades se incrementan en los de mayor edad, a excepción de jugar online que se mantiene en niveles parecidos durante todas las franjas etarias de la adolescencia.

Booker, Kelly y Sacker (2018) han observado que altos niveles de interacción en los medios sociales en la adolescencia temprana tiene mayores implicaciones para el bienestar de ellas. De acuerdo a Fernández de Arroyabe, Lazkano y Eguskiza (2018) también hay diferencias en el uso según el género: las mujeres interactúan intensamente, usando más YouTube para ver vídeos, mientras que los hombres prefieren las videoconsolas. Las mujeres consumen más información que los hombres en todos los temas, excepto en los deportes. En lo que afecta a la edad, Fietkiewicz, Lins, et al. (2016) observan la relación de esta variable con el uso de medios sociales en términos intergeneracionales.

\subsection{Usos y consecuencias}

Abundan los estudios sobre las consecuencias negativas del uso de redes sociales por los adolescentes. El número de perfiles en redes sociales entre usuarios de 14 a 17 años se relaciona con la hiperactividad, la falta de atención, la sensación de soledad y el Fear of Missing Out -FoMO- (Barry, Sidoti, et al., 2017). Este fenómeno les incita a acceder continuamente a las redes, perdiendo horas de sueño por la excitación cognitiva previa (Scott y Cleeland-Woods, 2018). En Instagram, Frison y Eggermont (2017) asocian la depresión de adolescentes a un incremento de publicaciones y Sheldon y Newman (2019) observan un aumento del uso de esta red entre los menores tímidos o rechazados para escapar de la realidad.

Desde la perspectiva de género, De Vries, Peter, et al. (2016) vinculan la intensidad de uso de redes con la insatisfacción de su físico. Malo-Cerrato, Martin-Perpiñá y Viñas Poch (2018) detectan excesos entre las chicas (11-18 años) relacionados a la inestabilidad emocional, el carácter impulsivo y una menor vinculación con el entorno familiar y académico. También son ellas quienes tienen mayor consciencia del uso problemático de Internet y de las redes sociales (Koronczai, Urbán y Demetrovics, 2020). Chang, Li, et al. (2019) sugieren que las comparaciones tienen efectos negativos para la autoestima física y es una práctica similar en hombres y mujeres (Ho, Lee y Liao, 2016) cuando tienen a las celebridades como reflejo para su propia imagen corporal. 
Otro de los problemas que observan Moreno y Uhls (2019) es la merma de la comunicación no verbal, tan importante para el desarrollo relacional del individuo. A ello se une la sensación de vigilancia que perciben los jóvenes a medida que se intensifica su acceso a las redes sociales (Ranzini y Hoek, 2017). Sin embargo, Young, Len-Ríos y Young (2017) concluyen que los efectos nocivos no solo dependen de la frecuencia de uso. También inciden otros factores como es precisamente la motivación. Marino, Vieno, et al. (2016) defienden que la cantidad de tiempo en Facebook no indica necesariamente un uso problemático. El consumo moderado y mantenido de redes sociales de la mayoría de menores (83\%) se asocia con niveles bajos de depresión, agresión y otros problemas (Coyne, Padilla-Walker, et al., 2019).

En menor medida, se registran estudios que aportan una perspectiva más optimista y redundan en las consecuencias positivas. Ziv y Kiasi (2016) observan un efecto beneficioso en el uso de Facebook entre adolescentes poco hábiles en las vías de comunicación tradicionales. Y Resnik y Bellmore (2019) constatan la adquisición de habilidades distintas en adolescentes según el carácter del medio (online/offline).

En el caso particular de los juegos online y videojuegos, probablemente su ejecución aporta más oportunidades de aprendizaje social que la televisión porque intensifican las relaciones con amigos (Bond, 2019). Por otra parte, el consumo de música y videoclips contribuye al desarrollo de su identidad, moldeando sus gustos y estableciendo relaciones a través de las redes sociales con usuarios de intereses afines (Pluretti y Bobkowski, 2019).

\subsection{Usos y motivaciones}

Otros estudios se centran en las motivaciones de uso y en los cambios en el comportamiento, sin examinar las consecuencias. En la adolescencia se adquiere autonomía, desplazando a la familia por las relaciones con iguales motivadas por la afinidad de intereses (Amanda, 2020; Moreno y Uhls, 2019) y la mutua influencia (Kindschi, Cieciuch, et al., 2019). Los adolescentes muestran una clara necesidad por socializar, reafirmarse y consolidar su autoestima (Valkenburg, Koutamanis y Vossen, 2017). Su aprecio por la tecnología permite ampliar sus relaciones y vías comunicativas. Los bruscos cambios experimentados en sus relaciones de amistad, variables y flexibles, entre los 11 y 17 años, (Felmlee, Macmillan, et al., 2016) se agudizan con la rápida transformación del entorno digital (Menezes, Arvanitidis, et al., 2019).

Otras investigaciones (García-Jiménez, López-de-Ayala y Montes-Vozmediano, 2020; García-Ruiz, Tirado y Hernando, 2017) afirman que las principales motivaciones para que los adolescentes utilicen las redes sociales son la consolidación de la identidad, la autopresentación, la popularidad y el reconocimiento de su entorno. Y Tulane, Vaterlaus y Beckert (2018) observan el uso de dispositivos como escudo ante situaciones en las que pretenden esquivar un encuentro cara a cara. 
Las fluctuaciones de comportamiento de los menores les hace establecer comparativas entre ellos mismos, los otros (influencers y celebridades) y sus pares conocidos o no (Kleemans, Daalmans, et al., 2016). Por ello, siguen más a las cuentas de estos perfiles que a las de sus familiares (Gil-Quintana y Fernández-Galiano, 2020) para confirmar sus propios gustos e intereses aumentando así la autoconfianza (Santamaría de la Piedra y Meana, 2017) y la necesidad de pertenencia a un grupo (Lai, Hsieh y Zhang, 2019).

Recibir una respuesta positiva de sus pares les motiva para publicar contenidos destinados a dar una autoimagen atractiva y ser apreciados (Yau y Reich, 2018). Esto sucede más entre las niñas que también tienden a pedir ayuda a sus pares para aumentar el interés en sus comunidades. La recurrencia en la reafirmación de su identidad se asocia con un uso intenso de la tecnología, en especial, de las redes (Candale, 2017). De acuerdo a Krasnova, Veltri, et al., (2017), las mujeres están principalmente motivadas por los usos relacionales, como el mantenimiento de lazos estrechos y el acceso a la información social en redes cercanas y lejanas. Y los hombres basan sus intenciones en su capacidad de obtener información de carácter general.

El presente trabajo analiza el comportamiento de los adolescentes de 12 a 16 años en las redes sociales para delimitar el consumo en función de las actividades que llevan a cabo teniendo en cuenta la edad y el género. Los objetivos específicos de investigación que se persiguen son los siguientes: a) determinar los usos de medios sociales por parte de adolescentes; b) precisar en qué medida la edad es un factor que influye en el uso y selección de medio social; c) delimitar si la variable de género afecta al uso y selección de plataforma de red social. Y las hipótesis iniciales de investigación que se plantean son:

H1) Los adolescentes, a diferente edad, presentan un consumo y elección de plataforma de red social diferentes, en lo que se refiere a las acciones de autopresentación, entretenimiento, relaciones sociales, búsqueda de contenidos y acceso a la información de actualidad.

H2) El comportamiento de los adolescentes en el consumo y determinación de medio social cambia en función del género, en lo que afecta a la autopresentación, el entretenimiento, las relaciones sociales, la búsqueda de contenidos y el acceso a la información de actualidad.

\section{Metodología}

Esta investigación se basa en una encuesta representativa $(n=515)$ de estudiantes de Educación Secundaria Obligatoria $\left(1^{\circ}\right.$ a $4^{\circ}$ de la ESO) de la Comunidad de Madrid, con edades entre 12 y 16 años, durante el curso académico 2019-2020. La muestra 
se propone como una aproximación suficiente, pero al mismo tiempo preliminar, al objeto de estudio, previa a futuras actuaciones investigadoras más amplias.

El diseño muestral procede de un muestro poliétapico estratificado por conglomerados. La estratificación se realizó en función de la titularidad del centro (público, privado o concertado) y tomando en consideración las diferencias entre Madrid capital y el resto de localidades de la Comunidad de Madrid. Los conglomerados correspondían a los centros educativos de ESO en un primer nivel (16 centros). Tras su correspondiente aceptación, se procedió a la selección de las unidades de análisis de la segunda etapa que estaban constituidas por las aulas. Los encuestados presentaron tanto sus autorizaciones como la de los padres o tutores en los casos así requeridos por la legislación. Para la realización de la encuesta se solicitó también permiso a las direcciones de los centros educativos. Toda la documentación requerida sigue la normativa española y europea de protección de datos. El análisis de error se fijó en $\pm 4,37 \%$ para los datos globales bajo el supuesto de $p=q=0,5$ con un nivel de confianza del 95\%, y bajo el supuesto de un muestreo aleatorio simple.

El trabajo de campo se desarrolló del 24 de octubre al 19 de diciembre de 2019. El acceso a la información se realizó por el sistema C.A.P.I. Las entrevistas se realizaron de forma personal en los colegios teniendo en cuenta cuotas de género, ámbito y curso. El tiempo de cumplimentación de la encuesta fue de alrededor de 25 minutos. Los ítems propuestos fueron de elaboración propia.

Los datos recogidos fueron ponderados manteniéndose las proporciones de la distribución real de la población según la distribución por tipo de municipio, tipo de centro, nivel educativo y género de los encuestados (según los datos de la Oficina de Estadística y Consejería de Educación de la Comunidad de Madrid). Para el análisis de los datos, y mediante el programa SPSS, se han realizado tablas de contingencia y las diferencias significativas se han situado en el nivel de validez estadística de $\chi^{2}<0.05$. Para este trabajo se han tenido en cuenta los ítems referidos al uso que se hace de los medios sociales con mayor consumo en el momento de la realización de la encuesta: Instagram, YouTube, Facebook, Snapchat y Twitter. Para la presentación de los resultados, se establecen cinco categorías de acciones o actividades, que engloban, agrupadas, las preguntas que se plantearon:

- Autopresentación (exposición de contenidos referidos al propio autor): a) «publicar/colgar contenidos en el propio perfil» y b) «actualizaciones sobre lo que hago o dónde estoy».

- Actividades de entretenimiento (formas digitales que les sirve para el esparcimiento y la distracción): a) «jugar online»; b) «seguir a mis actores, cantantes, deportistas e influencers favoritos»; c) «ver vídeos y música»; d) «buscar series, películas o programas de televisión»; e) «buscar con contenidos divertidos»; f) «buscar contenido sobre mis hobbies». 
- Relaciones sociales (establecimiento de contacto en diferentes formatos digitales): a) «ver vídeos o fotos de amigos o familiares»; b) «ver vídeos o fotos de desconocidos/consulta otros perfiles abiertos»; c) «hablar con amigos»; d) «hablar con familiares».

- Búsqueda de contenidos (rastreo por Internet de información que demandan sobre determinados asuntos): a) «contenido deportivo»; b) «información sobre ocio»; c) «información sobre famosos»; d) «información sobre salud»; e) «información de moda, belleza y estilismo»; f) «información comercial».

- Comportamiento en relación a la información de actualidad: a) «leer noticias de actualidad social o política», b) «compartir y comentar noticias».

\section{Resultados}

\subsection{Autopresentación en las plataformas de redes sociales}

Se observa diferencias a partir de la variable de género a la hora de "publicar/ colgar contenidos en el propio perfil» para YouTube y Snapchat. Así, los varones afirman en un 58,2\% que emplean YouTube para esta actividad frente al 30,6\% registrado entre ellas (Gráfico 1). En contraste y en relación con Snapchat, los chicos $(18,8 \%)$ tienen un uso más bajo que el registrado entre las encuestadas $(38 \%)$. Por su parte, la variable de edad tiene influencia solo para Instagram, con un uso superior al $90 \%$ en todos los casos, salvo para los de 12 años que se sitúa en casi 10 puntos por debajo.

Gráfico 1. Contingencia género/Publicación en YouTube de contenidos en su propio perfil.

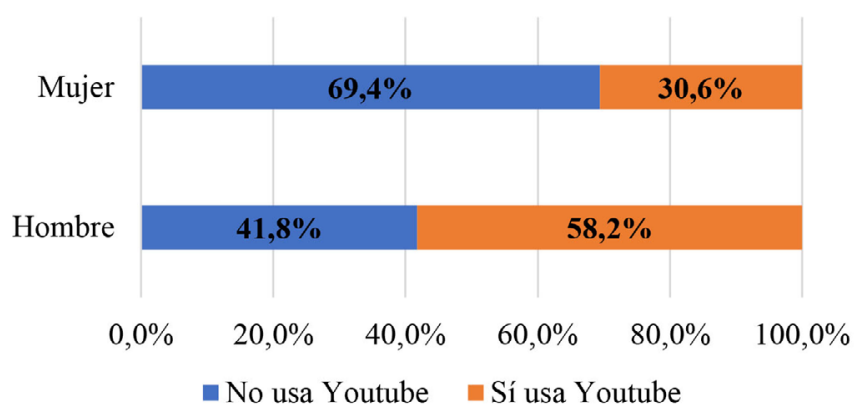

Fuente: elaboración propia 
Para las «actualizaciones sobre lo que hago o dónde estoy», se observa diferencias intensas en YouTube. Son más los adolescentes masculinos que lo consumen $(62,3 \%)$, mientras que ellas afirman recurrir a esta red en el 23,6\% de los casos. Resulta destacable que los hombres $(15,6 \%)$, pero sobre todo las mujeres apenas usan Twitter para este cometido (5,5\%). La edad no arroja diferencias significativas en este tipo de consumo para cualquiera de las redes analizadas.

\subsection{Medios sociales y relaciones sociales}

En relación a «ver vídeos o fotos de amigos o familiares», se observan fundamentalmente diferencias en YouTube en términos de género. De tal modo que es consumida por más de la mitad de los chicos $(56,5 \%)$ y menos de un tercio de las mujeres $(30,8 \%)$. Aunque con diferencias no tan pronunciadas, se produce el caso contrario en Snapchat: algo más de una cuarta parte de las mujeres se decanta por esta red frente al 16,4 por ciento de los encuestados, tendencia muy similar a la detectada para Twitter.

La edad sí tiene influencia en Instagram en esta actividad: De los 12 a los 15 años se produce una tendencia creciente de uso (de $71,4 \%$ a $95 \%$ ) pero cae hasta casi los valores originales (75\%) cuando alcanzan los 16 años. También se hallan datos significativos relacionados con el acceso a YouTube y en Facebook, pero la línea de tendencia es más irregular: cuando más lo usan es a los 12 y a los 13 para la primera y a los 13 y 16 en la segunda. Para Snapchat el receso secuencial es más evidente: los mayores valores son hallados entre los más pequeños. En cualquiera de los casos, a excepción de Facebook, los encuestados de 16 años marcan un menor interés por su entorno más cercano en redes sociales.

Para «ver vídeos o fotos de desconocidos/consulta otros perfiles abiertos» se observa en YouTube que el porcentaje de chicos que sí lo usa $(67,2 \%)$ es mayor que el de chicas (50\%). A su vez, un fenómeno a la inversa se produce con Twitter, aunque lo más destacable es el bajo uso de esta red social en general. La edad se presenta como un factor clave para entender el comportamiento en Instagram: la tendencia es creciente a medida que cumplen años, pero vuelven al mínimo del $80 \%$ que se registraba a los 12 cuando tienen 16; de este modo se vuelve a repetir la generalidad de que a esta edad máxima de los encuestados se detecta una menor curiosidad para consultar los contenidos de otros, ya sean desconocidos o de su entorno (Gráfico 2).

También se ha analizado el papel de las diferentes plataformas cuando se trata de «hablar con amigos». El género se presenta como un indicador interesante. Así, YouTube no aparece como una red especializada en esta tarea, especialmente entre ellas: $7,8 \%$ frente al $26,8 \%$ de los varones. Tampoco es recurrente esta actividad en Snapchat, pero menos para los varones (16\%), mientras que el 26,5\% de chicas sí lo emplea. Sí se observan diferencias relativas al impacto de la edad: 
En Instagram comienza y termina prácticamente en la misma proporción, y en los valores intermedios se observa una línea ascendente: un 67\% afirma acudir a esta red social para «hablar con amigos» a los 12 años. Este porcentaje sube hasta los $14(94,1 \%)$ y 15 años (92,7\%) para bajar al 66,7\% de los 16 años. En Twitter, hay una actividad baja, para este cometido: no superando el 5,9\% con la salvedad del 23,5\% que afirman emplear esta red a los 16 años. En Facebook la tendencia es similar, con cifras más bajas de uso.

Gráfico 2. Contingencia Edad/«ver vídeos o fotos de desconocidos/consulta otros perfiles abiertos» en Instagram.

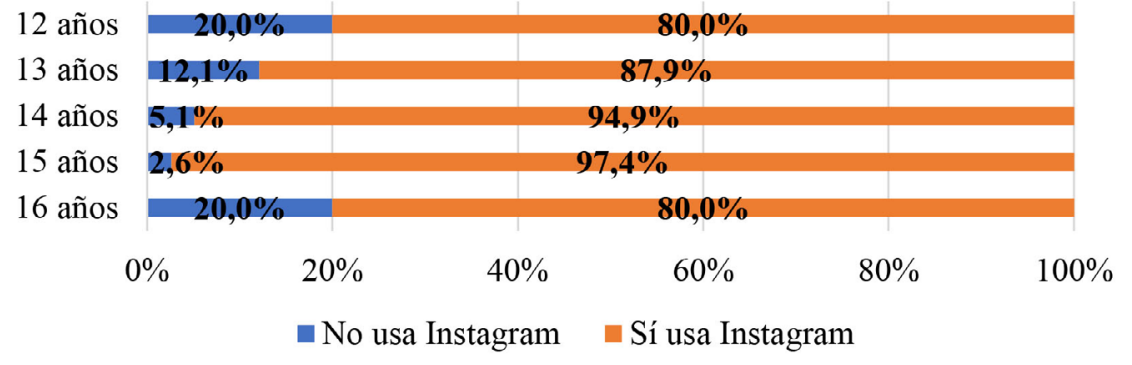

Fuente: elaboración propia

Finalmente, en lo que respecta a la actividad «hablar con familiares» se observa un acceso a Instagram más pronunciado de los varones 53,7\% del total de esta categoría mientras que ellas solo lo usan en el 37,4\% de los casos. En YouTube hay un uso bajo, sobre todo entre ellas, el 6,8\% dentro de su categoría frente al 22,9\% de los encuestados. Por otra parte, se detecta la influencia de la edad para el uso de Snapchat y Facebook. En la primera, y con cifras modestas en cualquier caso, se observa un acceso decreciente progresivo desde los 12 años, edad en la que el $16,3 \%$ confirma su acceso para esta acción, hasta el $0 \%$ a la edad de 16 años. Por su parte, los menores que recurren a Facebook representan (con algunas leves diferencias) un porcentaje bajo que no supera el 10,9\% (13 años), y que sube al $23,5 \%$, a los 16 , cuestión que vuelve a reforzar la inclinación por esta red cuando son más mayores, independientemente de la acción que desarrollen.

\subsection{Actividades de entretenimiento}

En el apartado de "ver vídeos y música», el género solo es estadísticamente significativo para YouTube, aunque no muy acusado: $88,6 \%$ de ellos lo usa y 95,4\% de ellas. Por otra parte, sí se observa la influencia de la edad en Instagram. De tal modo que desde el uso para esta actividad del 32,9\% en los 12 años, su utilización 
crece a los $13(50,8 \%)$, alcanza su máximo en los 14 años $(67,3 \%)$ se mantiene en cifras similares a los $15(65,8 \%)$ para finalmente caer al 57,9\% de encuestados con 16 años.

De acuerdo a los datos, la acción «jugar online» es más acusada en Instagram y en YouTube entre los chicos con una media de 35,7\% para ambas redes frente a una media de 16,2\% registrada entre ellas (Gráfico 3 ). No se han encontrado datos significativamente estadísticos $\left(\chi^{2}>0,05\right)$ en la edad. Para la búsqueda de contenido sobre sus hobbies, no hay diferencias significativas en ninguna red en relación al género. Sí para la edad y se repite la línea ascendente de los 12 a 15 para caer al mínimo aproximado de los 52 puntos porcentuales a los 16. Esta propensión generalizada parece indicar que aquellos que usan una determinada red desde las edades más bajas se mantienen fieles a ella a lo largo de su adolescencia; el resto la usa más como moda y por su efímera popularidad.

Gráfico 3. Contingencia Sexo/«jugar online» en Instagram y YouTube.

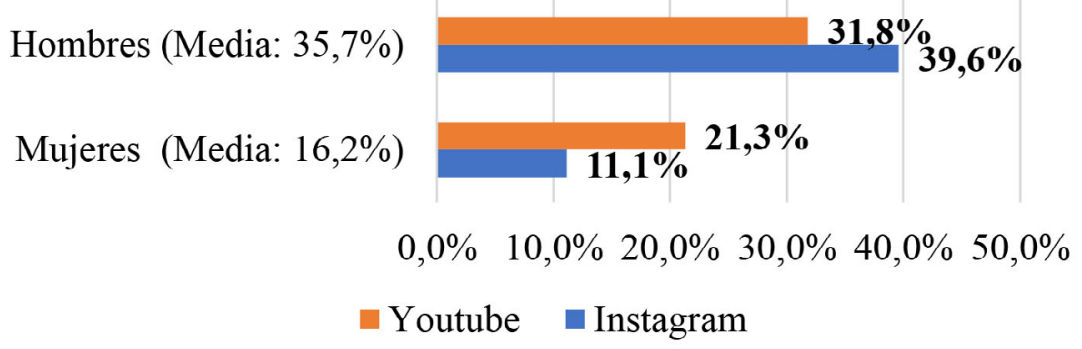

Fuente: elaboración propia

Para «buscar series, películas o programas de televisión», no se pueden establecer diferencias significativas estadísticas por género. La edad influye solo en Instagram. En términos generales, no muestra una utilización acusada. Este fenómeno es claro en los más pequeños (14,5\% en los 12 años) y luego, se presenta una curva ascendente $(13: 25,2 \% ; 14 ; 29,6 \% ; 15: 32,4 \%)$ para bajar nuevamente al menor uso a los 16 años, con un 12,5\% que afirma usar esta red social para este tipo de búsqueda.

Cuando se trata de «buscar contenidos divertidos», Instagram presenta un mayor uso masculino (suponen el 55,6\% del total). También se observa que es la única red social en que la edad interviene como factor relevante. De nuevo, comienza con unas cifras del $57,8 \%$ de los menores, pero el descenso aquí comienza a los 15 hasta un $61,1 \%$ a los 16 . 
El género no tiene influencia clara para «seguir a mis actores, cantantes, deportistas e influencers favoritos». Por su parte, la edad es importante en Instagram. En esta red, los menores se entusiasman por ella cada vez más a medida que son mayores y luego se repite la línea de bajada a los 16; no obstante, hay que matizar que la inclinación sigue siendo muy alta en toda la adolescencia; de hecho, el $80 \%$ de los más mayores acomete esta acción en Instagram. Por su parte, en Facebook no se observa ningún patrón claro, siendo muy variado y sin subir del 7,8\%.

\subsection{Búsqueda de contenidos}

En relación a «buscar contenido deportivo», y en lo que se refiere a Instagram, se advierte un mayor uso por parte de ellos $(71,3 \%)$ que de ellas, que lo utilizan en un 53,4\%. Datos similares ofrece YouTube: Un 75,4\% de chicos buscan contenido deportivo, en su franja de edad, frente al $50,5 \%$ de las encuestadas. En cualquier caso, solo representan el $26,1 \%$ del total. Por su parte, la edad solo afecta al consumo de Instagram. Sigue siendo sintomática la creciente de los 12 a los 15 para volver aproximadamente a los valores originales cuando alcanzan los 16 que solo es utilizada esta red para la búsqueda de estos contenidos por la mitad de los encuestados de esta edad.

Se detecta un uso mayor de Instagram para «buscar información sobre ocio» entre los varones. En términos globales, ellos representan el 56\% de quienes usan Instagram a tal efecto. También hay un consumo mayor de los chicos en YouTube (64,6\% de ellos y $53,8 \%$ de ellas). En Twitter, aunque con uso bajo (global de casi el 10\%), también es mayor para los varones. Para esta actividad, se detecta solo influencia de la edad en YouTube: El mayor acceso se da a los 12 años (74\%) pero en este caso y de forma prácticamente excepcional, la línea fluctúa aunque en torno a valores más bajos, con picos máximos, por este orden, en los 13 y en los 15 .

En cuanto a la actividad «buscar información sobre famosos», no hay diferencias estadísticas de género. Solo se ha encontrado influencia de la edad en Instagram. El uso más pronunciado se produce a los 14 años (88,7\%) (Gráfico 4). En realidad, se observa una campana de Gauss con una menor utilización en los 16 años $(56,3 \%)$ y los $12(60,5 \%)$. En una posición intermedia se encontrarían los de 13 $(75,7 \%)$ y 15 años $(83,7 \%)$.

«Buscar información sobre salud» no ofrece un gran seguimiento ni marcadas divergencias por género. A su vez, la variable edad muestra diferencias solo para Twitter. Destaca el hecho de que el 22,2\% de los menores con 12 años afirman emplear esta red social para informarse sobre salud. A partir de aquí, el uso es bajo y no supera el 8,3\%, cifra observada entre los encuestados de 16 años. 
Gráfico 4. Contingencia Edad/«Buscar información sobre famosos» en Instagram.

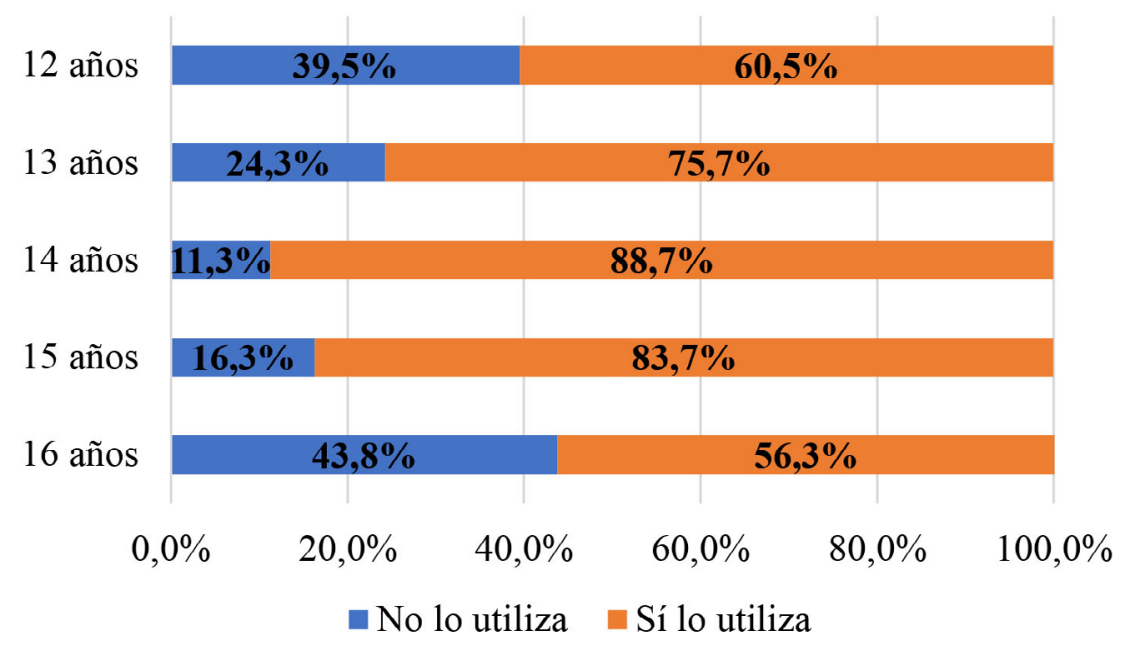

Fuente: elaboración propia

Con respecto a «buscar información de moda, belleza y estilismo», los datos más relevantes son los vinculados a YouTube. Aquí, las chicas representan casi las tres cuartas partes del total de adolescentes que ejercen esta acción. Por su parte, también la edad es un factor clave para entender el comportamiento en Instagram y YouTube. En el primer caso, se vuelve a ver un patrón ya conocido: Un 53,5\% del total de encuestados confirma que a los 12 años usa Instagram para esta actividad. Sube de los 13 a los 15 y baja los 16 años hasta el $60 \%$. Sin embargo, para YouTube, el uso más fuerte se observa en la edad más temprana con un $88,4 \%$, para ir descendiendo con posterioridad en todas las franjas etarias establecidas.

En la búsqueda de información comercial, los datos son significativos para Instagram: el $77,1 \%$ de los varones acceden a esta red, mientras que ellas lo hacen en un $66,9 \%$. La tendencia es similar para YouTube y Twitter. La edad es un factor influyente en el caso de YouTube, aunque sin un patrón claro. A los 12 y 15 años se registran los picos más altos ( $56 \%$ y $37,8 \%$ respectivamente) pero el descenso se repite y con mayor agudeza que en otros casos a los $16(14,3 \%)$.

\subsection{Información de actualidad}

El 58,8\% de los varones acceden a Instagram para «leer noticias de actualidad social o política», mientras que ellas lo hacen en un $45,7 \%$. Una de las claves es que 
es mayor el porcentaje de las chicas que no accede a la información de actualidad. Por otra parte, y aunque YouTube no sea ampliamente empleado para esta tarea, sigue siendo mayor entre la población masculina, tanto es así que casi se duplica (36,9\% de chicos frente al 19,7\% de chicas). Los datos extraídos de la encuesta indican que para «leer noticias de actualidad social o política», Instagram es la única red social que aporta datos significativos (Gráfico V). Parte con un porcentaje en la primera edad analizada del $27,5 \%$ para subir hasta los el $63,2 \%$ a los 15 y ya bajar a los 16 años $(31,3 \%)$ :

Gráfico 5. Contingencia género/consulta de actualidad en Instagram.

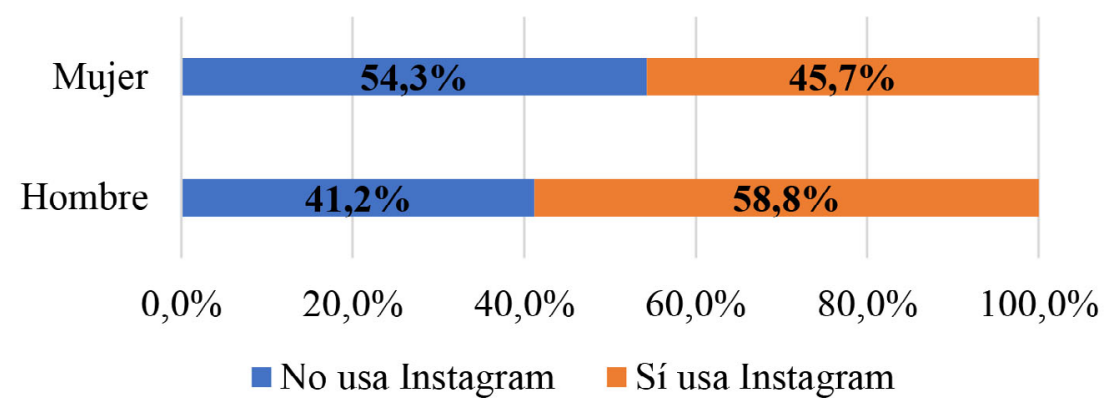

Fuente: elaboración propia

Con respecto a la acción de «compartir y comentar noticias» y en el caso de YouTube, tanto para hombres como mujeres, hay un uso establecido por debajo del $50 \%$. No obstante, ellos representan un $85,1 \%$ del total de personas que comparten y comentan noticias (un 30,8\% de varones, mientras que en el caso de ellas es del $5,8 \%)$. Estas cifras se repiten para Twitter, si bien de modo menos acusado. Así, los hombres representan el $71,9 \%$ frente al $28,1 \%$ de ellas. En relación a la edad, para Instagram, se observa otra vez el patrón ascendente hasta los 15 y registra el mínimo a los 16, esto último también se detecta en YouTube, pero en este caso, la tendencia de 12 a 15 no sigue una línea regular y son los más pequeños quienes usan más esta plataforma para esta actividad.

\section{Conclusiones y discusión}

La investigación realizada constata la preeminencia que los adolescentes otorgan a las redes sociales en sus comportamientos de consumo mediático. Coincidiendo con Menezes, Arvanitidis, et al. (2019), los datos constatan que la tecnología ya está instalada en las edades más tempranas (12-13 años). 
Las diferencias etarias han sido exploradas a partir de la primera hipótesis de trabajo. Destaca el uso masivo y preferente de Instagram en toda la muestra con un pico ostensible en los 12 años para tareas de autopresentación. Sin embargo, para la mayoría de actividades consideradas (redes/relaciones sociales; entretenimiento; buscar contenidos divertidos...) el uso de Instagram presenta un crecimiento progresivo y consonante con la edad, hasta los 16 años donde cae su uso. Los adolescentes mayores (16 años) presentan un cambio tímido, emergente, pero perceptible en el uso de Instagram. YouTube presenta la tendencia inversa: desciende con la edad, con la excepción de un pico a los 15 años. Snapchat es preferida por los menores y su uso decae con el incremento de edad, en la mayoría de actividades consideradas.

Los resultados confirman la modificación de los hábitos y preferencia de uso de los medios sociales en función de la edad (Fietkiewicz, Lins, et al., 2016; Tejada, Castaño y Romero, 2019). Uno de los hallazgos fundamentales de este trabajo radica en el consumo comparativo mayor de YouTube en las edades más tempranas. Por su parte, el consumo de Instagram aumenta con la edad (Civico, Gonzalez y Colomo, 2019). Este hecho se podría deber a que, en el marco de un uso más intensivo, se requiere una mayor capacidad para gestionar cada medio social.

Las posibles diferencias de género se tratan teniendo en cuenta la segunda hipótesis de trabajo. Los chicos prefieren YouTube para presentarse a sí mismos, para comentar lo que hacen, dónde están, explorar perfiles desconocidos o hablar con amigos. Jugar en línea es principalmente una actividad de los chicos, y existe una preferencia significativa relacionada con el género para esta actividad en todas las edades. La búsqueda de contenidos deportivos y las últimas noticias destaca entre los chicos a la hora de hacer una comparación de género. Las chicas triplican su actividad de compartir y comentar las noticias en todas las redes sociales estudiadas

La primera elección de las chicas es para las actividades de autopresentación y seguimiento de las actualizaciones de la familia y los amigos está en YouTube, aunque eligen esta red sólo la mitad de las veces que los chicos. Por el contrario, su uso de Snapchat y Twitter es el doble que el de los chicos para las mismas actividades. Estos resultados encajarían parcialmente con otros estudios (Fernández de Arroyabe, Lazkano y Eguskiza, 2018) en los que se demuestra una mayor inclinación por los juegos en lo que afecta a ellos (Garmendia, Jiménez, et al., 2019) y una mayor actividad social para las chicas. No obstante, se encuentra divergencias en cuanto al grado de consumo de YouTube. Y potencialmente un uso más diversificado. Los datos también estarían relacionados positivamente con las principales motivaciones de las mujeres (Krasnova, Veltri, et al., 2017), en términos de usos relacionales, mantenimiento de los lazos estrechos y el acceso a la información social en redes cercanas y lejanas. 
Por su parte, los chicos prefieren obtener información de carácter general. Pero esto ocurriría solo con algunos tipos de información. Ambos géneros comparten interés por la búsqueda de información sobre famosos; también coinciden en el desinterés por compartir y comentar noticias de actualidad. No existen diferencias de género en el uso de las redes sociales a la hora de buscar contenidos sobre hobbies, seguir celebridades o buscar información sobre las mismas, ni para información sobre salud.

Las actividades preferidas de los adolescentes en redes sociales presentan una enorme variedad, pero, en todos los casos, se identifica una serie de constantes: el culto extremo a la imagen en todas sus facetas, un comportamiento de búsqueda orientado en la afinidad con los intereses personales, el desinterés por la actualidad político/económica en contraste con un desmedido interés por el seguimiento de los más cercanos (familiares y amigos) o los más populares/famosos. Este último aspecto coincide con los resultados que revelan los estudios de Amanda (2020), Moreno y Uhls (2019), Cieciuch, et al. (2019) y Valkenbourg, Koutamanis y Vossen (2017). El uso de las redes sociales entre los adolescentes parece compartir ciertas características que superan las tradicionales diferencias asociadas al contexto geográfico o cultural.

Una de las limitaciones de este estudio es el tratamiento de otros medios sociales (pero que en este trabajo no se han identificado en concreto), aunque se presentan con un peso significativo en algunas respuestas. Por otra parte, el estudio de las variables consumo y actividad podría completarse con el estudio de las motivaciones o la satisfacción, para tener una visión de conjunto más exacta del complejo fenómeno estudiado. Las motivaciones podrían explicar las preferencias de consumo y la satisfacción obtenida, así como el tipo de experiencia diferenciada que posibilita cada red social.

Los adolescentes están instalados en el cambio constante y presenta comportamientos de consumo esquivos, a veces efímeros, lo que dificulta su investigación. Además, están muy influenciados por situaciones coyunturales como las modas, siempre que las prescriba alguien cercano. Crecen en un entorno caracterizado por la sobreinformación y el surgimiento permanente de nuevos medios/espacios de comunicación, con base tecnológica. En este entorno han nacido y se desenvuelven con soltura. Este estudio contribuye a entender la relación de los adolescentes con las redes sociales, sus preferencias y el tipo de prácticas que despliegan en la era de la convergencia mediática.

\section{Bibliografía}

Al-Menayes, Jamal J. (2015). Motivations for using social media: An exploratory factor analysis. En: International Journal of Psychological Studies, vol.7, $\mathrm{n}^{0} 1$. Toronto: CCSE, 43-50. doi: https://doi.org/10.5539/ijps.v7n1p43 
Amanda, Nabila Rizki (2020). Social Interaction Among Adolescents Who Use Social Media. En: Advances in Social Science, Education and Humanities Research, n ${ }^{\circ}$ 430. Ámsterdam: Atlantis Press, 112-117. doi: https://dx.doi.org/10.2991/ assehr.k.200120.025

Barry, Christopher T.; Sidoti, Chloe L.; Briggs, Shanelle M.; Reiter, Shari R. y Lindsey, Rebecca A. (2017). Adolescent social media use and mental health from adolescent and parent perspectives. En: Journal of adolescence, $n^{\circ} 61$. Ámsterdam: Elsevier, 1-11. doi: http://dx.doi.org/10.1016/j.adolescence.2017.08.005

Bergström, A. y Jervelycke Belfrage, M. (2018). News in social media: Incidental consumption and the role of opinion leaders. En: Digital Journalism, vol.6, ${ }^{0} 5$. Londres: Taylor \& Francis, 583-598. doi: https://doi.org/10.1080/21670811.2018 .1423625

Booker, Cara L.; Kelly, Yvonne J. y Sacker, Amanda (2018). Gender differences in the associations between age trends of social media interaction and well-being among 10-15 year olds in the UK. En: BMC Public Health, vol.18, $\mathrm{n}^{0} 1$. Londres: BioMed Central, 1-12.

Bond, Robert (2019). Social network determinants of screen time among adolescents. En: The Social Science Journal. Ámsterdam: Elsevier, . doi: https://doi. org/10.1016/j.soscij.2019.08.009

Candale, Carmen Valentina (2017). Las características de las redes sociales y las posibilidades de expresión abiertas por ellas. La comunicación de los jóvenes españoles en Facebook, Twitter e Instagram. En: Colindancias-Revista de La Red de Hispanistas de Europa Central, $\mathrm{n}^{\circ}$ 8. Timisoara: EUV, 201-218.

Chang, Leanne; Li, Pengxiand; Loh, Renae Sze Ming; Chua y Trudy Hui Hui (2019). A study of Singapore adolescent girls' selfie practices, peer appearance comparisons, and body esteem on Instagram. En: Body image, $\mathrm{n}^{\circ} 29$. Ámsterdam: Elsevier, 90-99. doi: https://doi.org/10.1016/j.bodyim.2019.03.005

Cívico Ariza, Andrea; González García, E. y Colomo Magaña, E. (2019). Análisis de la percepción de valores relacionados con las TIC en adolescentes. En: Espacios, vol.40, n ${ }^{0} 32$. Caracas: Talleres de Impresos Omar.

Coyne, Sarah M.; Padilla-Walker, Laura M.; Holmgren, Hailey G. y Stockdale, Laura A. (2019). Instagrowth: a longitudinal growth mixture model of social media time use across adolescence. En: Journal of research on adolescence, vol.29, $\mathrm{n}^{\circ} 4$. Hillsdale: Erlbaum, 897-907. doi: https://doi.org/10.1111/jora.12424

De Vries, Dian A.; Peter, Jochen; De Graaf, Hanneke y Nikken, Peter (2016). Adolescents' social network site use, peer appearance-related feedback, and body dissatisfaction: Testing a mediation model. En: Journal of youth and adolescence, vol.45, $\mathrm{n}^{\circ} 1$. Malden: Wiley, 211-224. doi: https://doi.org/10.1007/s10964-015-0266-4 
Felmlee, Diane H.; McMillan, C.; Rodis, P. I. y Osgood, D. W. (2018). The evolution of youth friendship networks from 6th to 12th grade: School transitions, popularity and centrality. En: Duane F. Alwin, Diane H. Felmlee y Derek A. Kreager (Eds.). Social networks and the life course. Cham: Springer.

Fernández de Arroyabe, Ainhoa; Lazkano, Iñaki; Eguskiza, Leire (2018). Consumo y creación audiovisual en euskera de los adolescentes gipuzkoanos: sobrevivir en un contexto digital dominado por lenguas hegemónicas. En: European Public \& Social Innovation Review, vol.3, $\mathrm{n}^{0} 1$. San Sebastián: Sinnergiak Social Innovation, 82-94.

Fietkiewicz, Kaja J.; Lins, Elmar; Baran, Katsiaryna S. y Stock, Wolfgang G. (2016). Inter-generational comparison of social media use: Investigating the online behavior of different generational cohorts. En: 2016 49th Hawaii International Conference on System Sciences (HICSS). Koloa: IEEE, 3829-3838.. doi: https:// doi.org/10.1109/HICSS.2016.477

Frison, Eline y Eggermont, Steven (2017). Browsing, Posting, and Liking on Instagram: The Reciprocal Relationships between Different Types of Instagram Use and Adolescents' Depressed Mood. En: Cyberpscyhology, Behavior, and Social Networking, vol.20, $\mathrm{n}^{\circ} 10$. Bethesda: NLM, 603-609. doi: https://doi.org/10.1089/ cyber.2017.0156

García-Jiménez, Antonio; López-de-Ayala López, María Cruz; Montes-Vozmediano, Manuel (2020). Características y percepciones sobre el uso de las plataformas de redes sociales y dispositivos tecnológicos por parte de los adolescentes. En: Zer, vol.25, nº48. Bilbao: UPV, 269-286. doi: https://doi.org/10.1387/zer.21556

García-Ruiz, Rosa; Tirado, Ramón; Hernando, Angel (2018). Redes sociales y estudiantes: motivos de uso y gratificaciones. Evidencias para el aprendizaje. En: Aula abierta, vol.47, no3. Oviedo: Universidad de Oviedo, 291-298. doi: https:// doi.org/10.17811/rifie.47.3.2018.291-298

Garmendia, Maialen; Jiménez, Estefanía; Karrera, Iñaki; Larrañaga, Nekane; Casado, Miguel Angel; Martínez, Gemma y Garitaonandia, Carmelo (2019). Actividades, Mediación, Oportunidades y Riesgos online de los menores en la era de la convergencia mediática. León: INCIBE.

Garmendia, Maialen; Jiménez, Estefanía; Casado, Miguel Ángel; Mascheroni, Giovanna (2016). Riesgos y oportunidades en internet y uso de dispositivos móviles entre menores españoles (2010-2015). Madrid: Red.es/Universidad del País Vasco.

Gil-Quintana, J. y Fernández-Galiano, M. (2020). Instagram posts, interactions, truths and lies of Spanish adolescents. En: Texto Livre: Linguagem e Tecnologia, vol.13, $\mathrm{n}^{0} 1$. Belo Horizonte: Universidade Federal de Minas Gerais.

Gini, Gianluca; Marino, Claudia; Xie, Jia-Yan; Pfetsch, Jan; Pozzoli, Tizziana (2019). Associations of Traditional and Peer Cyber-Victimization With Adolescents' Internet Use: A Latent Profile Analysis. En: Cyberpsychology: Journal of Psychoso- 
cial Research on Cyberspace, vol.13, nº4. Brno, Masaryk University. doi: https:// doi.org/10.5817/CP2019-4-1

Ho, Shirley S.; Lee, Edmund W. y Liao, Youquin (2016). Social network sites, friends, and celebrities: The roles of social comparison and celebrity involvement in adolescents' body image dissatisfaction. En: Social Media + Society, vol.2, n ${ }^{\circ} 3$, Londres: Sage, 1-11. doi:http://doi.org/10.1177/2056305116664216

Kindschi, Martin; Cieciuch, Jan; Davidov, Eldad; Ehlert, Alexander; Rauhut, Heiko; Tessone, Claudio J.; Algesheimer, René (2019). Values in adolescent friendship networks. En: Network Science, vol.7, nº4. Cambridge: Cambridge University Press, 1-25. doi: https://doi.org/10.1017/nws.2019.16

Kleemans, Mariska; Daalmans, Serena; Carbaat, Ilaa; Anschütz, Doeschka (2018). Picture perfect: the direct effect of manipulated Instagram photos on body image in adolescent girls. En: Media Psychology, vol.21, n ${ }^{0} 1$. Londres: Taylor \& Francis, 93-110. doi: https://doi.org/10.1080/15213269.2016.1257392

Koronczai, Beatrix; Urbán, Róbert; Demetrovics, Zsolt (2020). Parental bonding and problematic internet or social media use among adolescents. En: Psychiatria Hungarica: A Magyar Pszichiatriai Tarsasag tudomanyos folyoirata, vol.35, nº1. Budapest: Magy Pszichiatr. Tras., 73-80.

Krasnova, H.; Veltri, N. F.; Eling, N.; Buxmann, P. (2017). Why men and women continue to use social networking sites: The role of gender differences. En: The Journal of Strategic Information Systems, vol.26, no 4 . Ámsterdam: Elsevier, 261284. doi: https://doi.org/10.1016/j.jsis.2017.01.004

Lai, Hui-Min; Hsieh, Pi-Jung; Zhang, Ren-Cheng (2019). Understanding adolescent students' use of Facebook and their subjective wellbeing: a genderbased comparison. En: Behaviour \& information technology, vol.38, $\mathrm{n}^{\circ} 5$. Londres: Taylor \& Francis, 533-548. doi: http://dx.doi.org/10.1080/0144929X.2018.1543452

Len-Ríos, María E.; Hughes, Hilary E.; McKee, Laura G. y Young, Henry N. (2016). Early adolescents as publics: A national survey of teens with social media accounts, their media use preferences, parental mediation, and perceived Internet literacy. En: Public Relations Review, vol.42, nº1. Ámsterdam: Elsevier, 101-108. doi: http://dx.doi.org/10.1016/j.pubrev.2015.10.003

Malo-Cerrato, Sara; Martín-Perpiñá, Maria-de-las-Mercedes y Viñas-Poch, Ferran (2018). Uso excesivo de redes sociales: Perfil psicosocial de adolescentes españoles. En: Comunicar, vol.26, no56, 101-110. doi: https://doi.org/10.3916/C56-2018-10 Marcelino Mercedes, Georgina Victoria (2015). Spanish youth and teenagers migrating through social networks. From Tuenti to Facebook and from Facebook to Instagram. The second migration. En: Icono 14. Revista Científica de Comunicación y Tecnologías, vol.13, no2. Madrid: Icono 14, 48-72. doi: https://doi.org/10.7195/ ri14.v13i2.821 
Marino, Claudia; Vieno, Alessio; Pastore, Massimiliano; Albery, Ian P.; Frings, Daniel y Spada, Marcantonio M. (2016). Modeling the contribution of personality, social identity and social norms to problematic Facebook use in adolescents. En: Addictive behaviors, vol.63. Ámsterdam: Elsevier, 51-56. doi: http://dx.doi. org/10.1016/j.addbeh.2016.07.001

Menezes Marluci; Arvanitidis Paschalis; Smaniotto Costa, Carlos y Weinstein, Zvi (2019). Teenagers' Perception of Public Spaces and Their Practices in ICTs Uses. En: Smaniotto Costa, Carlos, et al. (eds). CyberParks - The Interface Between People, Places and Technology. Springer: Cham

Moreno, Megan A. y Uhls, Yalda T. (2019). Applying an affordances approach and a developmental lens to approach adolescent social media use. En: Digital health, vol.5. Londres: Sage, 1-6. doi: https://doi.org/10.1177\%2F2055207619826678

Murphy, Gráinne; Corcoran, Ciara; Tatlow-Golden, Mimi; Boyland, Emma y Rooney, Brendan (2020). See, Like, Share, Remember: Adolescents' Responses to Unhealthy-, Healthy-and Non-Food Advertising in Social Media. En: International Journal of Environmental Research and Public Health, vol.17, ${ }^{\circ}$ 7. Basilea: MDPI, 2181. doi: https://doi.org/10.3390/ijerph17072181

Pluretti, Roseann y Bobkowski, Piotr S. (2019). Social media, adolescent developmental tasks, and music. En: McFerran, Katrina; Derrington, Philippa y Saarikallio, Suvi (eds.). Handbook of Music, Adolescents, and Wellbeing.

Ranzini, Giulia y Hoek, Elles (2017). To you who (I think) are listening: Imaginary audience and impression management on Facebook. En: Computers in Human Behavior, vol.75. Ámsterdam: Elsevier, 228-235. doi: http://dx.doi.org/10.1016/j. chb.2017.04.047

Resnik, Felice y Bellmore, Amy (2019). Connecting online and offline social skills to adolescents' peer victimization and psychological adjustment. En: Journal of youth and adolescence, vol.48, $\mathrm{n}^{\circ} 2$. Cham: Springer, 386-398. doi: https://doi. org/10.1007/s10964-018-0953-z

Santamaría de la Piedra, Elena y Meana, Rufino J. (2017). Redes sociales y fenómeno influencer. Reflexiones desde una perspectiva psicológica. En: Miscelánea Comillas. Revista de Ciencias Humanas y Sociales, vol.75, $\mathrm{n}^{0} 147$. Madrid: Universidad Pontifica de Comillas, 443-469.

Scott, Holly y Cleland-Woods, Heather (2018) Fear of missing out and sleep: cognitive behavioural factors in adolescents' nighttime social media use. En: Journal of Adolescence, vol.68. Ámsterdam: Elsevier, 61-65. doi: https://doi.org/10.1016/j. adolescence.2018.07.009

Sheldon, P. y Newman, M. (2019). Instagram and American Teens: Understanding Motives for Its Use and Relationship to Excessive Reassurance-Seeking and Interpersonal Rejection. En: The Journal of Social Media in Society, vol.8, $n^{0} 1$. , 1-16. 
Tejada Eneko; Castaño, Carlos; Romero, Ainara (2019). The habits of use in social networks of preadolescents. En: RIED. Revista Iberoamericana de Educación a Distancia, vol.22, n ${ }^{\circ}$ 2. Madrid: UNED, 119-133. doi: http://dx.doi.org/10.5944/ ried.22.2.23245

Throuvala, Melina A.; Griffiths, Mark D.; Rennoldson, Mike y Kuss, Daria J. (2018). Motivational processes and dysfunctional mechanisms of social media use among adolescents: A qualitative focus group study. En: Computers in Human Behavior, vol.93. Ámsterdam: Elsevier, 164-175. doi: https://doi.org/10.1016/j. chb.2018.12.012

Trültzsch-Wijnen, C. W.; Trültzsch-Wijnen, S.; Siibak, A. (2015). Using and not using social media: what triggers young people’s SNS practices? En: Zeller, Frauka; Ponte, Cristina y O’Neill, Brian (eds.) Revitalising Audience Research: Innovations in European Audience Research. Nueva York: Routledge.

Tulane, Sarah; Vaterlaus, J. Mitchell y Beckert, Troy E. (2018). A mixed methods examination of adolescents' reasons for pretending to text. En: Journal of adolescence, vol.69. Ámsterdam: Elsevier, 175-179. doi: https://doi.org/10.1016/j. adolescence.2018.10.003

Twenge, J.M.; Martin, G.N.y Spitzberg, B.H. (2019). Trends in US Adolescents' media use, 1976-2016: The rise of digital media, the decline of TV, and the (near) demise of print. En: Psychology of Popular Media Culture, vol.8, $\mathrm{n}^{\mathrm{o}} 4$. Washington: APA, 329-345. doi: http://dx.doi.org/10.1037/ppm0000203

Valkenburg, Patti M.; Koutamanis, Maria; Vossen, Helen G. (2017). The concurrent and longitudinal relationships between adolescents' use of social network sites and their social self-esteem. En: Computers in human behavior, $\mathrm{n}^{0} 76$. Ámsterdam: Elsevier, 35-41. doi: http://dx.doi.org/10.1016/j.chb.2017.07.008

Van Dam, Sophia y Van Reijmersdal, Eva (2019). Insights in adolescents' advertising literacy, perceptions and responses regarding sponsored influencer videos and disclosures. En: Cyberpsychology: Journal of Psychosocial Research on Cyberspace, vol.13, nº2. Brno: Masaryk University. doi: http://dx.doi.org/10.5817/CP2019-2-2 van Eldik, Anne; Kneer, Julia y Jansz, Jeroen (2019). Urban \& online: social media use among adolescents and sense of belonging to a super-diverse city. En: Media and Communication, vol.7, n ${ }^{0} 2$. Lisboa: Cogitatio, 242-253. doi: http://dx.doi. org/10.17645/mac.v7i2.1879

Vannucci, Anna; Simpson, Emily G.; Gagnon, Sonja y Ohannessian, Christine McCauley (2020). Social media use and risky behaviors in adolescents: a metaanalysis. En: Journal of Adolescence, vol.79. Ámsterdam: Elsevier, 258-274. doi: https://doi.org/10.1016/j.adolescence.2020.01.014

Yau, Joanna C. y Reich, Stephanie M. (2019). "It's Just a Lot of Work”: Adolescents' Self-Presentation Norms and Practices on Facebook and Instagram. En: 
Journal of Research on Adolescence, $\mathrm{n}^{0} 29$. Malden: Wiley, 196-209. doi: https:// doi.org/10.1111/jora.12376

Young, Rachel; Len-Ríos, María y Young, Henry (2017). Romantic motivations for social media use, social comparison, and online aggression among adolescents. En: Computers in Human Behavior, $\mathrm{n}^{0} 75$. Ámsterdam: Elsevier, 385-395. doi: http:// dx.doi.org/10.1016/j.chb.2017.04.021

Ziv, Ido y Kiasi, Mali (2016). Facebook's contribution to well-being among adolescent and young adults as a function of mental resilience. En: The Journal of Psychology, vol.150, $\mathrm{n}^{\circ} 4$. Londres: Taylor \& Francis, 527-541. doi: https://doi.or $\mathrm{g} / 10.1080 / 00223980.2015 .1110556$ 